\title{
The Aryl Hydrocarbon Receptor Nuclear Translocator (ARNT/HIF-1 $\beta$ ) is Influenced by Hypoxia and Hypoxia-Mimetics
}

\author{
Matthias Wolffa,b Wolfgang Jelkmann ${ }^{a}$ Jürgen Dunst ${ }^{b}$ Reinhard Depping ${ }^{a}$ \\ aInstitut für Physiologie, Center for Structural and Cell Biology in Medicine, Universität zu Lübeck, \\ bKlinik für Strahlentherapie, UK-SH Campus Lübeck, Lübeck
}

\author{
Key Words

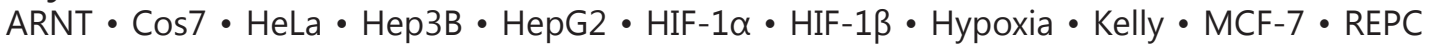 \\ $\cdot$ T47D
}

\begin{abstract}
Background: The Aryl Hydrocarbon Receptor Nuclear Translocator (ARNT, HIF-1ß) is a member of the basic-Helix-Loop-Helix PER/ARNT/SIM (bHLH/PAS) protein family and a vital transcriptional regulator regarding development and physiological adaptation processes. ARNT is discussed to be linked with cancer, and other diseases. ARNT is known to be translocated into the cell nucleus, where accumulation of the protein takes place. ARNT is a heterodimerisation partner of the xenobiotic ligand activated Aryl Hydrocarbon Receptor $(\mathrm{AhR})$, the Single Minded proteins (SIM), the cardiovascular helix-loop-helix factor 1 and the Hypoxia Inducible Factor proteins (HIF- $\alpha$ ). ARNT is obligatory for HIF-1, HIF-2 and HIF-3 binding to DNA. Whereas degradation of the HIF- $\alpha$ subunits is suppressed by hypoxia, ARNT is generally regarded as constitutively expressed in excess within the cell, and stabilisation is commonly thought to be oxygen-independent. However, we provide evidence that the regulation of ARNT is far more complex. The aim of our study was to reevaluate the regulation of ARNT expression. Methods: We examined cell lines of different origin like MCF-7 and T47D (human breast cancer), HeLa (human cervix carcinoma), Hep3B and HepG2 (human hepatoma), Kelly (human neuroblastoma), REPC (human kidney) and Cos7 (primary primate kidney) cells. We used immunoblot analysis, densitometry, RT-PCR and transient transfection. Results and Conclusion: Our results show that ARNT protein levels are influenced by hypoxia and hypoxia mimetics such as cobalt(II)-chloride $\left(\mathrm{CoCl}_{2}\right)$ and dimethyloxalylglycine (DMOG) in a cell line specific manner. We demonstrate that this effect might be triggered by HIF- $1 \alpha$ which plays an important role in the process of stabilizing ARNT in hypoxia.
\end{abstract}




\section{Introduction}

The Aryl Hydrocarbon Receptor Nuclear Translocator (ARNT) is a member of the basicHelix-Loop-Helix PER/ARNT/SIM (bHLH/PAS) protein family and a crucial transcriptional regulator regarding organogenesis and neural development as well as physiological adaptation processes to hypoxia and pollutants [1, 2]. Beside ARNT (HIF-1 $\beta$ ), two more isoforms ARNT2 and ARNT3 (BMAL1, MOP3, JAP3, ARNTL1) exist in various species. ARNT and ARNT2 share a $>90 \%$ amino acid identity. ARNT has a more ubiquitous expression pattern than ARNT2 in adult tissue. Recent studies report a linkage of ARNT to several cell dysfunctions and diseases, such as cancer and diabetes [3-5].

Like all bHLH-molecules, ARNT needs to dimerise to form active DNA binding complexes. ARNT is known to form heterodimers with the xenobiotic ligand activated Aryl Hydrocarbon Receptor (AhR) with whom metabolizing enzymes are induced, with HIF- $\alpha$ subunits (Hypoxia Inducible Factors) under low oxygen levels, SIM (Single Minded) proteins for neural development and CHF1 (cardiovascular helix-loop-helix factor 1) [6, 7]. We formerly showed, that ARNT is most likely translocated into the nucleus by classical nuclear import mediated by importins $\alpha / \beta$ [8]. Despite of a coexisting nuclear export ARNT accumulates mainly in the nucleus [9].

In hypoxia the $\alpha$-subunits of the Hypoxia Inducible Factors (HIF- $\alpha$ ) are stabilized and form heterodimers with ARNT (HIF-1 $\beta$ ) that regulate the cellular response to low oxygen levels. Three different isoforms of oxygen- dependent HIF- $\alpha$ subunits are currently identified: HIF-1 $\alpha$, HIF- $2 \alpha$ (EPAS1) and HIF-3 $\alpha$ [10]. In normoxic conditions HIF- $\alpha$ proteins undergo hydroxylation by Prolyl-Hydroxylase-Domain enzymes 1-3 (PHD1-3). Prolyl-hydroxylated HIF- $\alpha$ serves as a recognition motif for polyubiquitination by Von Hippel-Lindau Protein (pVHL) and subsequent proteasomal degradation. However ARNT lacks the Oxygen Dependent Degradation Domain (ODDD) and is considered to be constantly expressed in normoxic conditions $[9,11]$. It is current opinion that hypoxia has no effect on the ARNT levels [9, 11-14]. However scattered studies exist observing partially concomitant increase of ARNT protein levels in hypoxia [15-17]. Here we provide evidence that ARNT expression is indeed influenced and modulated depending on the cell type evaluated. This suggests that tumor cells might lose the ability of regulating ARNT during tumorigenesis. Our data show that the regulation of ARNT expression is far more complex than generally thought.

In our study we investigated the expression of ARNT under different types of hypoxia, hypoxia mimetics and Hypoxia Inducible Factors revealing cell line specific and cofactor dependent expression patterns.

\section{Materials and Methods}

Cell Culture, Hypoxic Induction and Hypoxia Mimetics

The cell lines MCF-7 (human breast ductal carcinoma), T47D (human breast ductal carcinoma), Hep3B (human hepatocellular carcinoma), HeLa (human cervix adenocarcinoma), HepG2 (human hepatocellular carcinoma) and Cos-7 (primate fibroblast-like kidney cells) were incubated in DMEM culture medium (Gibco). The cell lines REPC (primary human kidney, [18]) and Kelly (human neuroblastoma) were cultured in RPMI-1640 medium (Gibco). The culture medium was supplemented with $10 \%$ fetal calf serum (FCS, Gibco) and $100 \mathrm{IU} / \mathrm{ml}$ penicillin / 100 $\mathrm{g} / \mathrm{ml}$ streptomycin, respectively. The cells were grown in a humidified atmosphere at $37^{\circ} \mathrm{C}, 5 \% \mathrm{CO}_{2}$ and $20.9 \% \mathrm{O}_{2}$ (normoxia). For hypoxic studies, cells were placed in a humidified atmosphere at $37^{\circ} \mathrm{C}, 5 \% \mathrm{CO}_{2}$, balanced $\mathrm{N}_{2}$ with either $1 \%$ or $3 \% \mathrm{O}_{2}$. Cobalt(II)-chloride $\left(\mathrm{CoCl}_{2}\right)$ was used in a $50 \mu \mathrm{M}$ dilution and dimethyloxalylglycine (DMOG) was used in a $1 \mathrm{mM}$ dilution as hypoxia mimetic.

\section{Transient Transfection}

The cell lines MCF-7, T47D and Hep3B were transfected with siRNA using Lipofectamine ${ }^{\text {TM }}$ RNAiMAX transfection reagent (Invitrogen). Cells were grown to 50\% confluence in 6-well culture plates before transfection was done following manufacturer's protocol. Prior to the application of hypoxia the culture medium was changed and cells were incubated another 6 hours in normoxic conditions. 
Fig. 1. Immunoblot analysis of ARNT protein levels in MCF-7. Whole cell lysates (50 $\mu \mathrm{g})$ were analysed. Cells were cultured in normoxic $\left(21 \% \mathrm{O}_{2}\right)$ conditions followed by either 24 hours (Hox1\% 24h) or 48 hours (Hox $1 \% 48 \mathrm{~h})$ of hypoxic $\left(1 \% \mathrm{O}_{2}\right)$ induction. The hypoxic induction of ARNT protein levels are shown in comparison to their normoxic (Nox) controls measured by densitometry. Mean $\pm \mathrm{SD} ;{ }^{*} \mathrm{P}<0.05 ;{ }^{* *} \mathrm{P}<0.01$; ${ }^{* * *} \mathrm{P}<$ 0.001 (two-tailed P-value; $\mathrm{n}=6-11$ ). Means are depicted in percent. A representative immunoblot is shown. HIF- $1 \alpha$ protein levels serve as a control of hypoxic induction. Lamin $\mathrm{A} / \mathrm{C}$ serves as loading control.

Immunoblot Analysis and Densitometry

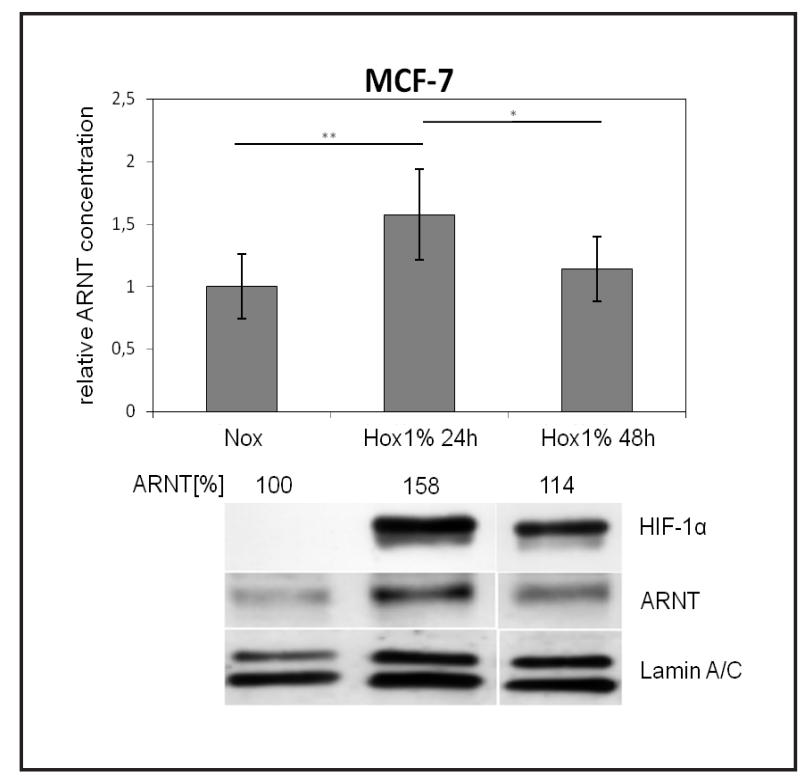

For Western blotting cells were collected after treatment, washed with ice-cold PBS, extracted with urea lysis buffer and sonicated gathering whole cell lysates. Protein concentrations were determined using Bio-Rad DC Protein Assay following manufacturer's protocol. Protein extracts were electrophoresed by SDS-PAGE and transferred to nitrocellulose membranes by semi-dry electroblotting. Membranes were blocked using 5\% non-fat milk in PBS. Primary antibodies (HIF-1 $\alpha$ : BD transduction, 1:1000; ARNT: Novus 1:1000) were incubated overnight followed by HRP corresponding secondary antibodies (DAKO 1:5000) added for 2 hours. ECL (GE Healthcare) was used as detection reagent. Protein quantity was compared using Aida Image Analyser v.4.27 (Raytest) according to the protocols. Each Western blot background was subtracted from the individual measuring area. Corresponding Lamin A/C loading was regarded $100 \%$ and the proportional value was calculated.

\section{RNA Isolation and RT-PCR}

To measure specific quantitative mRNA levels, total RNA was isolated from the cells using ABI Prism 6100 Nucleic Acid PrepStation (Applied Biosystems) following manufacturer's instructions. Then 0.2 $\mu \mathrm{g}$ of RNA were reverse transcribed using Superscript III (Invitrogen). The generated cDNA was used as template in quantitative real-time PCR analysis (RT-PCR). RT-PCR was performed using ABI 7000 Sequence Detection System (Applied Biosystems), TaqMan Universal PCR Master Mix (Applied Biosystems) and KAPA SYBR FAST Universal (Peqlab). Amplification was carried out using TaqMan Gene Expression Assays (Hs01100353_m1, Applied Biosystems) according to manufacturer's protocols. Primers specific for L28 housekeeping gene (sense: 5'ATGGTCGTGCGGAACTGCT-3` and reverse: $3^{\prime}$-TTGTAGCGGAAGGAATTGCG-5') were used for normalisation. Cycling conditions were an initial polymerase activation step at $95^{\circ} \mathrm{C}$ for 10 min, followed by 45 cycles at $95^{\circ} \mathrm{C}$ for $15 \mathrm{~s}, 55^{\circ} \mathrm{C}$ for $30 \mathrm{~s}$ and at $72^{\circ} \mathrm{C}$ for $20 \mathrm{~s}$. Samples were analysed in quadruplicate and relative amounts of mRNA were calculated using the $\Delta \Delta \mathrm{CT}$ method.

\section{Statistics}

Statistical analysis was performed with GraphPad InStat Software. Unpaired t-test was applied. Graphs are shown as means \pm standard deviation (SD). MTT survival assays were performed (data not shown) to ensure cell survival did not differ among samples.

\section{Results}

ARNT expression is induced in hypoxia in MCF-7, HeLa, Hep3B and REPC cells

We examined the quantity of ARNT protein levels by growing MCF-7 cells in normoxic $\left(21 \% \mathrm{O}_{2}\right)$ conditions for 24 hours followed by either 48 hours of normoxia (Nox) or 24 hours 
Fig. 2. Time dependent Immunoblot analysis of ARNT protein in MCF-7. Whole cell lysates (50 $\mu \mathrm{g})$ were analysed. Cells were cultured in hypoxic $\left(1 \% \mathrm{O}_{2}\right)$ conditions for $12 \mathrm{~h}, 24 \mathrm{~h}, 48 \mathrm{~h}, 72 \mathrm{~h}$ and $96 \mathrm{~h}$. Culture medium was exchanged daily, using $1 \% \mathrm{O}_{2}$ equilibrated medium. ARNT protein levels were measured by densitometry and are displayed in percent compared to $12 \mathrm{~h}$ hypoxia $1 \%$ (H1\% 12h). Lamin A/C serves as loading control.
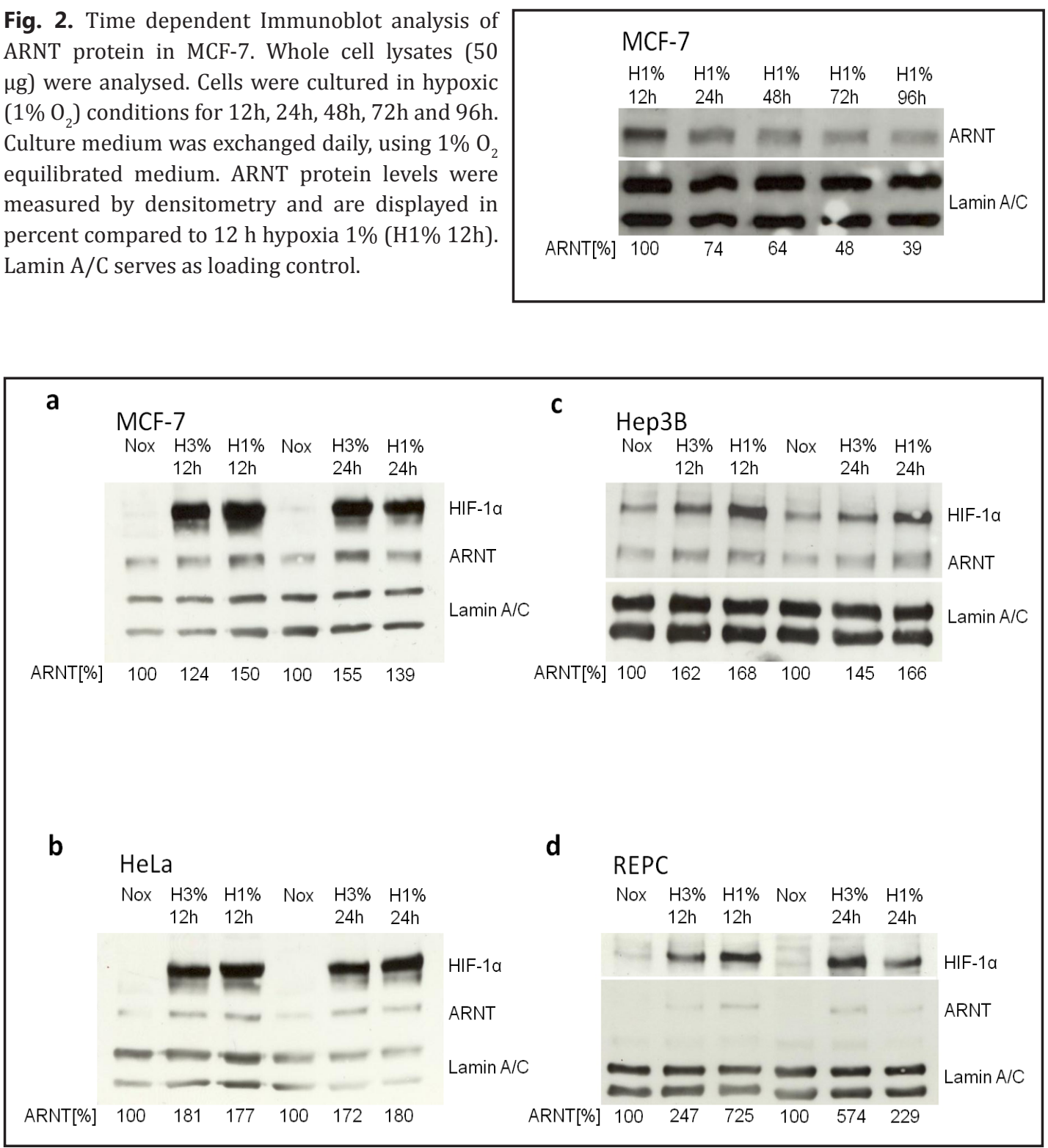

Fig. 3. Immunoblot analysis of ARNT protein levels in MCF-7, HeLa, Hep3B and REPC. Whole cell lysates $(50 \mu \mathrm{g})$ were analysed. Cells were cultured in hypoxic conditions $\left(3 \% \mathrm{O}_{2}\right.$ or $\left.1 \% \mathrm{O}_{2}\right)$ for 12 hours or 24 hours (H3\% 12h, H1\% 12h, H3\% 24h, H1\% 24h). ARNT protein levels were measured by densitometry and are displayed in percent to the normoxic controls (Nox). HIF-1 $\alpha$ protein levels serve as a control of hypoxic induction. Lamin A/C serves as loading control.

of normoxia and 24 hours of hypoxia $1 \%$ (Hox $1 \% 24 \mathrm{~h}, 1 \% \mathrm{O}_{2}$ ) or only 48 hours of hypoxia $1 \%$ (Hox 1\% 48h, 1\% $\mathrm{O}_{2}$ ). Whole cell extracts were analysed by immunoblotting using a monoclonal mouse anti-ARNT antibody. HIF-1 $\alpha$ protein was observed to confirm hypoxic cell induction. As shown in Fig. 1, ARNT protein levels were elevated in response to hypoxia in MCF-7 cells. 24 hours of $1 \%$ hypoxia incubation led to a significant increase of ARNT in comparison with 48 hours of $1 \%$ hypoxia.

MCF-7-cells were incubated up to 96 hours in 1\% hypoxia, to reveal long term effects of hypoxia. MCF-7 cells showed an increasing ARNT concentration after 12 hours. In further exposure to hypoxia ARNT concentrations began to decline to a baseline value (Fig. 2). 


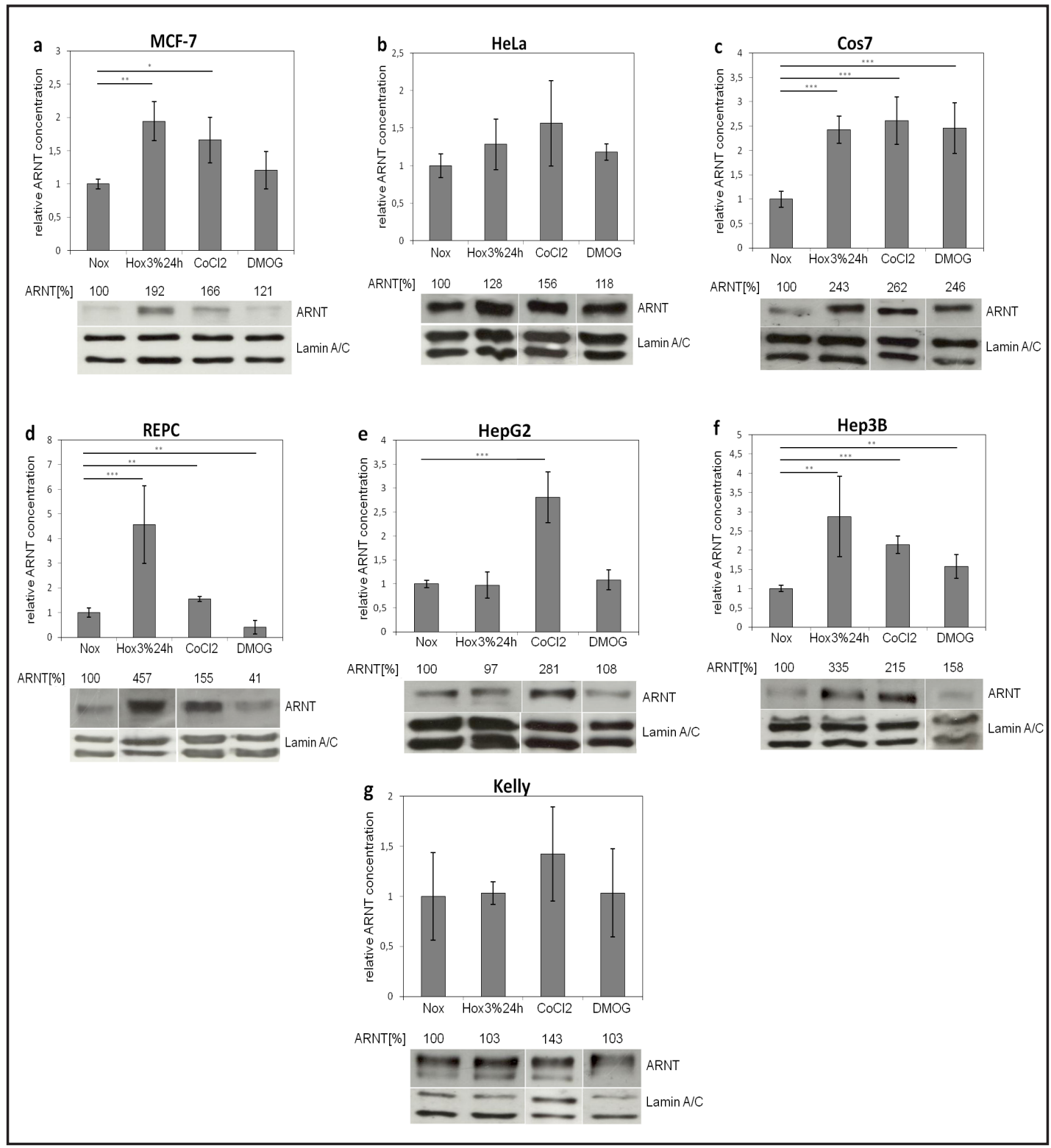

Fig. 4. Immunoblot analysis of ARNT protein levels in MCF-7, HeLa, Cos7, REPC, HepG2, Hep3B, and Kelly. Whole cell lysates $(50 \mu \mathrm{g})$ were analysed. Cells were cultured in normoxic $\left(21 \% \mathrm{O}_{2}\right)$ conditions (Nox), hypoxic $\left(3 \% \mathrm{O}_{2}\right.$ ) conditions for 24 hours (Hox 3\% 24h), normoxic conditions with $50 \mu \mathrm{M}$ cobalt(II)-chloride ( $\mathrm{CoCl} 2)$ and in normoxic conditions with $1 \mathrm{mM}$ dimethyloxalylglycine (DMOG). The induction of ARNT protein levels are shown in comparison to their normoxic (Nox) controls measured by densitometry. Mean $\pm \mathrm{SD} ;{ }^{*} \mathrm{P}<0.05 ;{ }^{* *} \mathrm{P}<0.01 ;{ }^{* * *} \mathrm{P}<0.001$ (two-tailed $\mathrm{P}$-value; $\mathrm{n}=3-7$ ). Means are shown in percent. A representative immunoblot is shown. Lamin A/C serves as loading control.

ARNT induction due to different intensities of hypoxia was measured culturing MCF-7 cells in $3 \%$ hypoxia $\left(3 \% \mathrm{O}_{2}\right)$ and $1 \%$ hypoxia $\left(1 \% \mathrm{O}_{2}\right)$ for 12 hours and 24 hours (Fig. 3a).

To elucidate differences between distinct cell lines we cultured HeLa, Hep3B and REPC cells similar to MCF-7 cells (Fig. 3b-d). In all hypoxia treated cell types ARNT protein levels were elevated. $1 \%$ hypoxia led to faster increase of ARNT protein levels but also to earlier normalisation than $3 \%$ hypoxia. 3\% hypoxia shows a maximal induction of ARNT after 24 hours. 


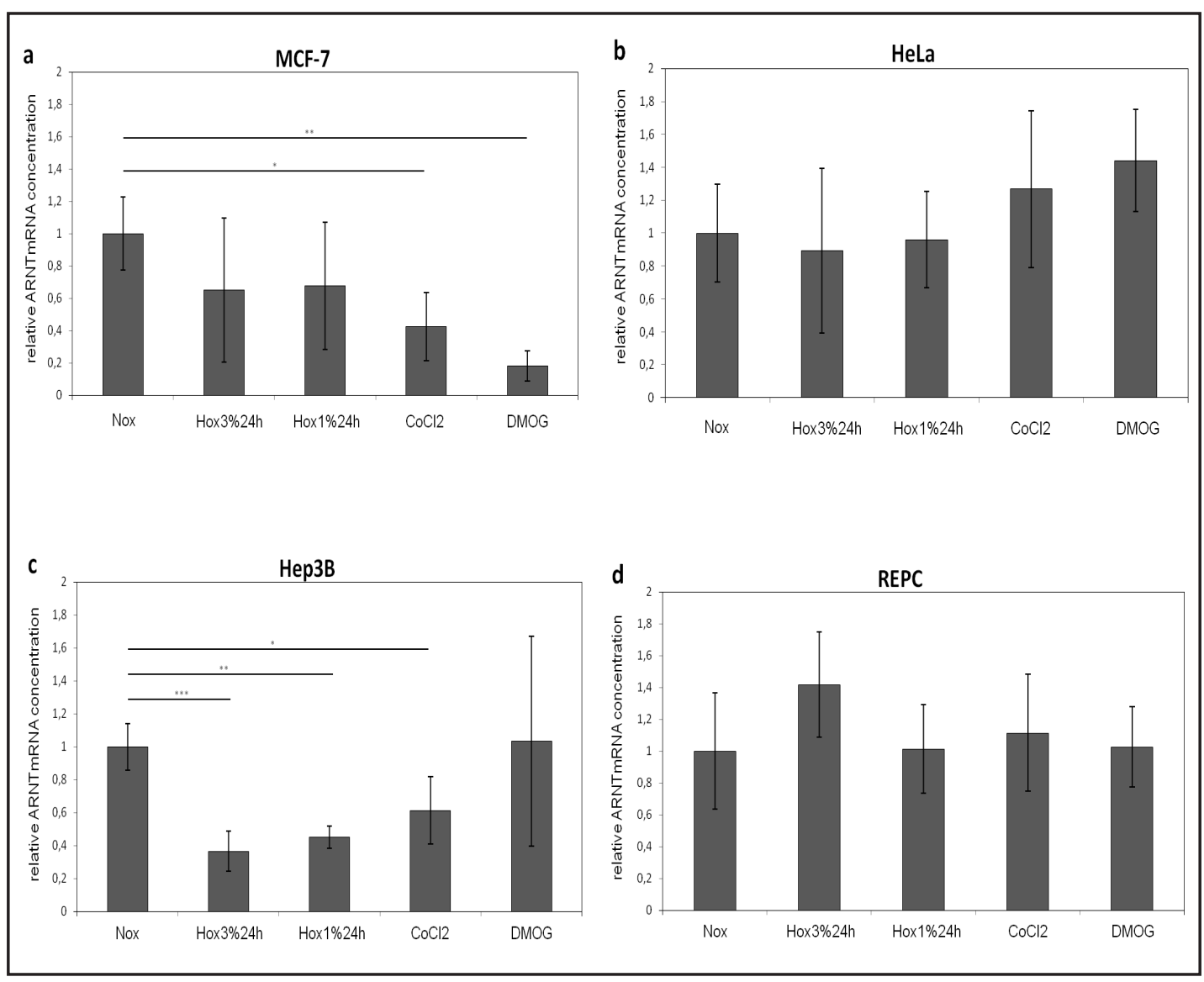

Fig. 5. Quantitative RT-PCR of ARNT mRNA levels in MCF-7, HeLa, REPC and Hep3B cells. Cells were cultured in normoxic $\left(21 \% \mathrm{O}_{2}\right)$ conditions followed by either 24 hours of normoxia (Nox), 24 hours of 3\% hypoxia (Hox 3\% 24h), 24 hours of 1\% hypoxia (Hox 1\% 24h), $50 \mu \mathrm{M} \mathrm{CoCl}_{2}$ for 24 hours $\left(\mathrm{CoCl}_{2}\right)$ or $1 \mathrm{mM}$ DMOG for 24 hours (DMOG). L28 housekeeping gene was used for normalisation. Relative amounts of mRNA were calculated using the $\Delta \Delta$ CT method. The diagram shows means of relative amounts of ARNT mRNA levels in comparison to normoxia (Nox). Mean $\pm \mathrm{SD}$; ${ }^{*} \mathrm{P}<0.05$; ${ }^{* *} \mathrm{P}<0.01$; ${ }^{* *} \mathrm{P}<0.001$ (two-tailed $\mathrm{P}$-value; $\mathrm{n}=4$ ).

Different cell lines show various reactions to hypoxia and hypoxia mimetics regarding ARNT protein levels

To investigate a relation between ARNT levels and HIF- $\alpha$, MCF-7, HeLa, Cos7, REPC, HepG2, Hep3B, and Kelly cells were treated with the hypoxia mimetics cobalt(II)-chloride $\left(\mathrm{CoCl}_{2}\right)$ and dimethyloxalylglycine (DMOG) (Fig. 4a-g).

HepG 2 and Kelly cells did not respond to 24 hours of hypoxia with an increase of ARNT protein levels. HeLa cells showed a slight ARNT induction in hypoxia and under treatment with hypoxia mimetics, but this effect was not statistically significant. MCF-7 cells clearly showed an increase of ARNT protein levels under 24 hours of $3 \%$ hypoxia and an increase of ARNT protein levels due to $\mathrm{CoCl}_{2}$ treatment. On the other hand DMOG treatment had no impact on ARNT levels in MCF-7 cells, although DMOG is a very potent HIF stabilizer. Cos7 cells showed a strong effect of ARNT induction to hypoxia as well as to the hypoxia mimetics $\mathrm{CoCl}_{2}$ and DMOG. REPC cells showed a strong response to hypoxia, however $\mathrm{CoCl}_{2}$ induction of ARNT was relatively small. DMOG reduced ARNT protein levels in REPC cells. HepG2 cells did not react with ARNT protein level induction to hypoxia nor to DMOG. However, HepG2 cells showed a very strong ARNT induction by $\mathrm{CoCl}_{2}$. Hep3B cells reacted with an increase of ARNT protein levels to all treatments. Kelly cells did not show any reaction in ARNT protein levels, neither to hypoxia nor to hypoxia mimetics. 


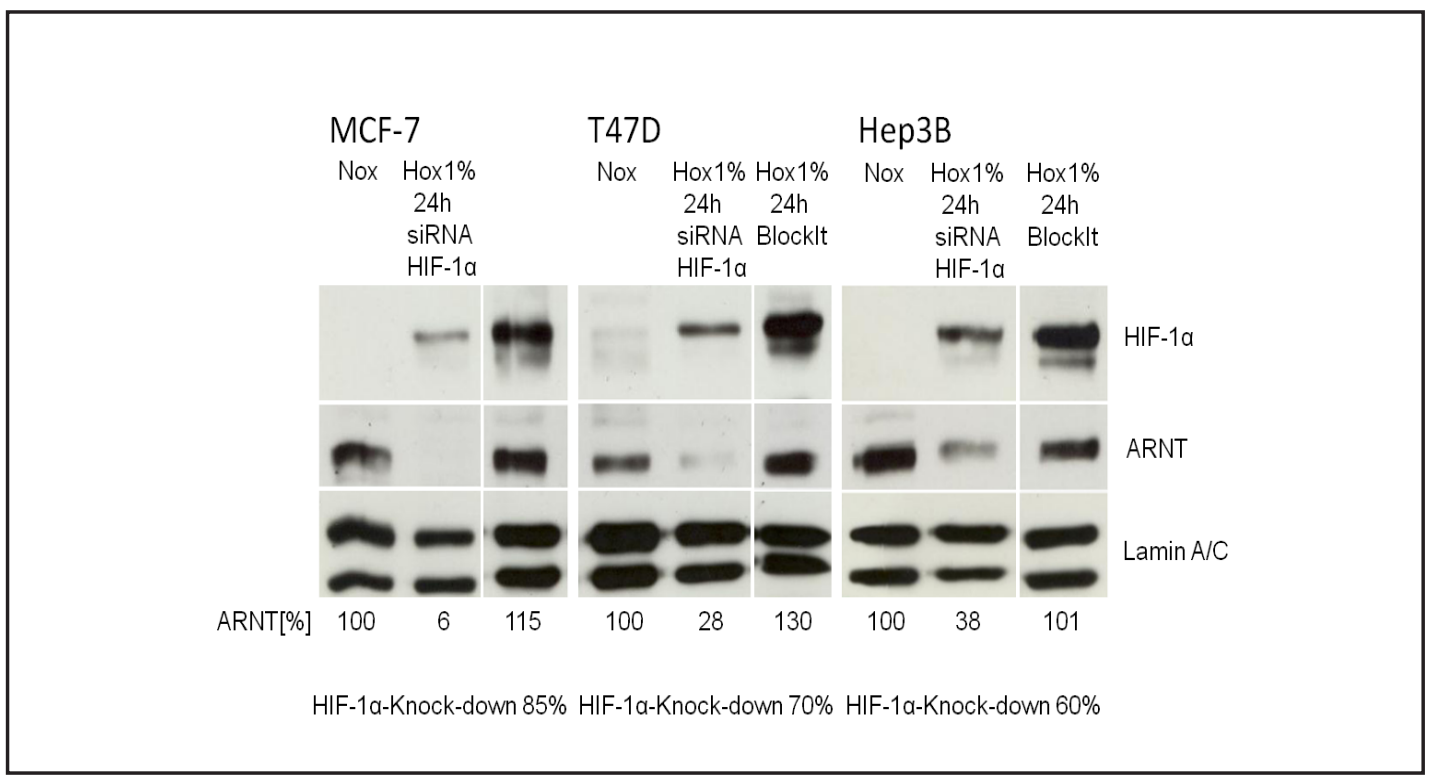

Fig. 6. Immunoblot analysis of ARNT protein levels in siRNA treated MCF-7, T47D and Hep3B. Whole cell lysates $(50 \mu \mathrm{g})$ were analysed. Cells were cultured in normoxic $\left(21 \% \mathrm{O}_{2}\right)$ conditions and transiently transfected using Lipofectamine ${ }^{\mathrm{TM}}$ RNAiMAX transfection reagent (Invitrogen) and HIF-1 $\alpha$ siRNA (Hox $1 \% 24 \mathrm{~h}$ siRNA HIF-1a) or BlockIt ${ }^{\mathrm{TM}}$ (Invitrogen) siRNA (Hox1\% 24h siRNA BlockIt) as a negative control for HIF-1 $\alpha$ specific knock down. ARNT protein levels were measured by densitometry and are displayed in percent. HIF-1 $\alpha$ protein levels serve as control for hypoxic induction and Hif- $1 \alpha$ knock down. Lamin A/C serves as loading control. $(n=4)$.

Changes in ARNT mRNA levels do not correlate with ARNT protein levels under the influence of hypoxia and hypoxia mimetics

We used the quantitative RT-PCR method, to examine mRNA levels related to the observed changes of ARNT protein levels. MCF-7, HeLa, REPC and Hep3B cells were grown in normoxic conditions before 3\% hypoxia, $1 \%$ hypoxia, $\mathrm{CoCl}_{2}$ and DMOG treatment for 24 hours was applied (Fig. 5). MCF-7 cells demonstrated an insignificant decline of ARNT mRNA levels in response to hypoxia. However a significant reduction of ARNT mRNA levels due to $\mathrm{CoCl}_{2}$ and DMOG treatment was observed. Whilst HeLa and REPC cells did not show any effect on ARNT mRNA levels after treatments, Hep3B cells reacted to hypoxia with a strong reduction of ARNT mRNA. A slighter diminishing reaction to $\mathrm{CoCl}_{2}$ was observed in these cells.

\section{ARNT protein is stabilized in the presence of HIF-1 $\alpha$}

MCF-7, T47D and Hep3B cells were transiently transfected with HIF-1 $\alpha$ siRNA, to clarify the effect of Hif- $1 \alpha$ on ARNT protein levels (Fig. 6). Cells were incubated for 48 hours in normoxia with transfection reagent RNAiMAX and siRNA followed by a 6 hour recovery phase. Subsequently cells were kept in $1 \%$ hypoxia for 24 hours. We observed that ARNT protein levels decreased in hypoxia if HIF-1 $\alpha$ was blocked. However, unspecific siRNA transfection did not influence the observed increase of ARNT protein levels in hypoxia.

\section{Discussion}

The Aryl Hydrocarbon Receptor Nuclear Translocator (ARNT/HIF-1 $\beta$ ) plays a role as a pivotal transcriptional regulator in cellular homeostasis. It functions as a heterodimerisation partner of various major regulator proteins, such as HIF- $\alpha$, SIM proteins, AhR and CHF1. 
Therefore it is involved in plenty of different regulatory cellular pathways [19]. Due to the fact that ARNT is thought to be constitutively expressed and not influenced by physiological or pharmacological changes, for example hypoxia, ARNT was not considered to be the limiting factor and a possible therapeutic target in earlier reports [9, 12-14, 20]. However, recent studies report a link between ARNT and several diseases, such as cancer [5]. HIF-1 $\alpha$ and HIF-2 $\alpha$ are well known to have crucial functions in cancer development and/or therapy [21]. As their mandatory cofactor, ARNT depletion would result in inactive or decreased levels of HIF- $\alpha$ complexes. To further underline the relevance of ARNT, we demonstrate for the first time that hypoxia, hypoxia mimetics and HIF-1 $\alpha$ play an important role in the regulatory mechanisms of ARNT expression depending on the cell type evaluated.

We examined eight different cell lines of 2 different host species to demonstrate the influence of hypoxia and hypoxia mimetics on ARNT protein expression. MCF-7 breast cancer cells react to hypoxia with an increase of ARNT protein levels, whereas ARNT mRNA levels seem to decrease or remain unchanged. This implies that a reciprocal feedback regulation exists between protein stabilisation and de novo synthesis. Similar down and up-regulations of gene transcription in hypoxia are known for several enzymes and molecules [21,22]. Our findings are not necessarily a result of a general reduction of mRNA synthesis in the cell as an answer to cell stress, because transcription of ESR2 gene is induced in hypoxia and HIF-1 $\alpha$ knock-down (unpublished data).

The exposure to hypoxia for a longer period than 48 hours leads to a reversion to basal ARNT protein levels which can be seen in normoxia. A similar peak of expression is known for HIF-1 $\alpha$ protein after 4 to 12 hours under hypoxic treatment. Further hypoxic incubation leads to declining HIF- $1 \alpha$ levels until reaching a steady state [15]. This fact could suggest a stabilizing effect of HIF- $1 \alpha$ overexpression towards ARNT. The formation of HIF-1 $\alpha$ / ARNT complexes in the nucleus could prevent ARNT degradation via proteasomes, which is in line with Choi et al. and Mandl et al. [20,23]. To investigate our findings we transiently knocked down HIF-1 $\alpha$ in MCF-7 cells and observed up to $90 \%$ reduction of ARNT protein levels in hypoxia compared to normoxia. This effect could also be seen in T47D and Hep3B cells. A cross reaction of HIF-1 $\alpha$ siRNA knocking down ARNT because of a strong sequence homology between ARNT and HIF- $\alpha$ was ruled out by in silico analysis of the siRNA and gene sequences. These findings suggest a strong correlation between ARNT and HIF- $1 \alpha$ protein expression. An explanation could be a stabilizing effect of the $\mathrm{O}_{2}$ independent ARNT binding partners, like the AhR, SIM and CHF1, towards ARNT in normoxia. In hypoxia, reduced levels of AhR, SIM and CHF1, could be compensated by overexpression of HIF- $\alpha$ regarding ARNT stabilisation. Knocking down HIF- $1 \alpha$ could therefore lead to highly decreased ARNT-levels via reduced de novo synthesis and less stability towards ubiquitination and proteasomal degradation, because dimerisation partners are lacking [20]. This hypothesis would underline a regulation of ARNT highly depending on binding partners, which would be in line with Choi et al. and Mandl et al. [20,23]. To examine, whether HIF- $\alpha$ induction leads to a concomitant increase of ARNT expression, we treated cells with $\mathrm{CoCl}_{2}$ and DMOG. Despite the fact that the HIF- $\alpha$ stabilizing effect of $\mathrm{CoCl}_{2}$ and DMOG is analogous, MCF-7 cells after DMOG react less strong with an increase of ARNT protein than after $\mathrm{CoCl}_{2}$ treatment. These observations are in line with a HIF- $\alpha$-stabilizing effect on ARNT. An opposite behavior between $\mathrm{CoCl}_{2}$ inducing ARNT in contrast to DMOG could be seen in HepG2 cells. Interestingly REPC cells react with a decrease of ARNT levels under DMOG treatment. Although the HIF- $\alpha$ stabilizing effect of DMOG is similar to $\mathrm{CoCl}_{2}$, a different cellular reaction by DMOG is not unusual due to a different mode of action. On the other hand Cos7 and Hep3B cells react to hypoxia and both hypoxia mimetics with a strong increase of ARNT protein levels, which is in line with the HIF- $\alpha$ stabilising effect postulated by Mandl et al. [23]. The fact that, in contrast to various tumor cell lines, ARNT levels are well influenced in the primary cell line Cos7 by hypoxia and hypoxia mimetics could imply a loss of function during tumorigenesis in certain tumors. Data regarding ARNT in cellular cancer development are scarce and therefore further investigation is needed. 
The Hep3B cell line shows significant increases of ARNT protein levels due to all three hypoxic treatments, whilst hypoxia and $\mathrm{CoCl}_{2}$ are reducing ARNT mRNA significantly. These findings support the hypothesis of a reciprocal feedback mechanism, which can be also hypothesised in MCF-7 cells. The REPC kidney cell line shows a deviant behavior. These cells react with a strong increase of ARNT protein due to hypoxia, but with a less intense induction due to $\mathrm{CoCl}_{2}$ and in dissent with declining ARNT protein levels under DMOG treatment as discussed above. But further REPC cells do not show any variance in ARNT mRNA levels under the various treatments implicating a regulation on protein basis.

Kelly cells show stable ARNT protein levels. Neither hypoxia nor hypoxia mimetics are capable of influencing ARNT. Such stable ARNT levels can be seen in HepG2 cells in hypoxia and DMOG treatment too, but this cell line reacts with a strong increase of ARNT protein upon $\mathrm{CoCl}_{2}$ treatment. HeLa cells do not show a significant change of ARNT levels, neither regarding protein nor mRNA. These findings support the present opinion, that ARNT is constantly expressed and not influenced by hypoxia as reviewed by Semenza [11]. We and others have shown that this view cannot be generalised [23]. We herewith provide data that contradict the hypothesis of a constantly expressed and unregulated ARNT heterodimerisation partner $[9,11-14]$ but which is in line with Chilov et al., [17]. Also supporting Choi et al. and Mandl et al., we show that the heterodimerisation with HIF- $\alpha$ is an important mechanism of stabilizing ARNT protein in hypoxia [20,23].

In conclusion, our data suggest a general stabilizing regulation of ARNT binding partners, like HIF- $\alpha$, AhR, SIM and CHF1 regarding ARNT expression. Furthermore, we can show that each cell line / type distinctly reacts to hypoxia and hypoxia mimetics regarding the induction ARNT protein synthesis. Therefore, an universalized cell spanning predication of ARNT protein expression is not permissible. These findings show that ARNT regulation should be further investigated, especially regarding tumorigenesis, because ARNT regulation is far more complex than appreciated today.

\section{Acknowledgments}

We are grateful to T. Svensson, B. Rudzewski and A.-K. Hellberg for excellent technical support. The authors declare that they have no conflict of interest.

\section{References}

1 Kewley RJ, Whitelaw ML, Chapman-Smith A: The mammalian basic helix-loop-helix/PAS family of transcriptional regulators. Int J Biochem Cell Biol 2004;36:189-204.

2 Shimoda LA, Semenza GL: HIF and the lung: role of hypoxia-inducible factors in pulmonary development and disease. Am J Respir Crit Care Med 2011;183:152-156.

-3 Nakabayashi H, Ohta Y, Yamamoto M, Susuki Y, Taguchi A, Tanabe K, Kondo M, Hatanaka M, Nagao Y, Tanizawa Y: Clock-controlled output gene Dbp is a regulator of Arnt/Hif-1ß gene expression in pancreatic islet $\beta$-cells. Biochem Biophys Res Commun 2013;434:370-375.

-4 Labrecque MP, Prefontaine GG, Beischlag TV: The aryl hydrocarbon receptor nuclear translocator (ARNT) family of proteins: Transcriptional modifiers with multi-functional protein interfaces. Curr Mol Med 2013;13:1047-1065.

-5 Liang Y, Li W-W, Yang B-W, Tao Z-H, Sun H-C, Wang L, Xia JL, Qin LX, Tang ZY, Fan J, Wu WZ: Aryl hydrocarbon receptor nuclear translocator is associated with tumor growth and progression of hepatocellular carcinoma. Int J Cancer 2012;130:1745-1754.

6 Shi S, Yoon DY, Hodge-Bell K, Huerta-Yepez S, Hankinson O: Aryl hydrocarbon nuclear translocator (hypoxia inducible factor 1beta) activity is required more during early than late tumor growth. Mol Carcinog 2010;49:157-165. 
Wolff/Jelkmann/Dunst/Depping: ARNT Influenced by Hypoxia

7 Chin MT, Maemura K, Fukumoto S, Jain MK, Layne MD, Watanabe M, Hsieh CM, Lee ME: Cardiovascular basic helix loop helix factor 1 , a novel transcriptional repressor expressed preferentially in the developing and adult cardiovascular system. J Biol Chem 2000;275:6381-6387.

8 Depping R, Steinhoff A, Schindler SG, Friedrich B, Fagerlund R, Metzen E, Hartmann E, Köhler M: Nuclear translocation of hypoxia-inducible factors (HIFs): involvement of the classical importin alpha/beta pathway. Biochim Biophys Acta 2008;1783:394-404.

-9 Berchner-Pfannschmidt U, Frede S, Wotzlaw C, Fandrey J: Imaging of the hypoxia-inducible factor pathway: insights into oxygen sensing. Eur Respir J 2008;32:210-217.

10 Lisy K, Peet DJ: Turn me on: regulating HIF transcriptional activity. Cell death Differ 2008;15:642-649.

-11 Semenza GL: Hypoxia-inducible factors in physiology and medicine. Cell 2012;148:399-408.

12 Kallio PJ, Pongratz I, Gradin K, McGuire J, Poellinger L: Activation of hypoxia-inducible factor 1alpha: posttranscriptional regulation and conformational change by recruitment of the Arnt transcription factor. Proc Natl Acad Sci USA 1997;94:5667-5672.

13 Huang LE, Gu J, Schau M, Bunn HF: Regulation of hypoxia-inducible factor 1alpha is mediated by an 02-dependent degradation domain via the ubiquitin-proteasome pathway. Proc Natl Acad Sci USA 1998;95:7987-7992.

14 Kaelin WG: Proline hydroxylation and gene expression. Annu Rev Biochem 2005;74:115-128.

15 Wang GL, Jiang BH, Rue EA, Semenza GL: Hypoxia-inducible factor 1 is a basic-helix-loop-helix-PAS heterodimer regulated by cellular $\mathrm{O}_{2}$ tension. Proc Natl Acad Sci USA 1995;92:5510-5514.

16 Iyer N V, Kotch LE, Agani F, Leung SW, Laughner E, Wenger RH, Gassmann M, Gearhart JD, Lawler AM, Yu AY, Semenza GL: Cellular and developmental control of $\mathrm{O}_{2}$ homeostasis by hypoxia-inducible factor 1 alpha. Genes Dev 1998;12:149-162.

17 Chilov D, Camenisch G, Kvietikova I, Ziegler U, Gassmann M, Wenger RH: Induction and nuclear translocation of hypoxia-inducible factor-1 (HIF-1): heterodimerization with ARNT is not necessary for nuclear accumulation of HIF-1alpha. J Cell Sci 1999;112:1203-1212.

18 Frede S, Freitag P, Geuting L, Konietzny R, Fandrey J: Oxygen-regulated expression of the erythropoietin gene in the human renal cell line REPC. Blood 2011;117:4905-4914.

19 Gu YZ, Hogenesch JB, Bradfield CA: The PAS superfamily: sensors of environmental and developmental signals. Annu Rev Pharmacol Toxicol 2000;40:519-561.

20 Choi H, Chun Y-S, Kim S-W, Kim M-S, Park J-W: Curcumin inhibits hypoxia-inducible factor-1 by degrading aryl hydrocarbon receptor nuclear translocator: a mechanism of tumor growth inhibition. Mol Pharmacol 2006;70:1664-1671.

21 Rankin EB, Giaccia AJ: The role of hypoxia-inducible factors in tumorigenesis. Cell Death Differ 2008;15:678-685.

22 Wenger RH, Rolfs A, Marti HH, Bauer C, Gassmann M: Hypoxia, a novel inducer of acute phase gene expression in a human hepatoma cell line. J Biol Chem 1995;270:27865-27870.

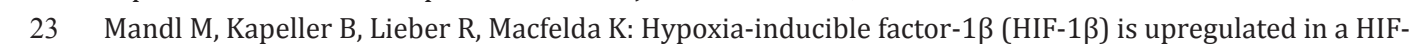
$1 \alpha$-dependent manner in 518A2 human melanoma cells under hypoxic conditions. Biochem Biophys Res Commun 2013;434:166-172. 